\title{
Plasma elafin, cathelicidin, and $\alpha$-defensins are increased in paediatric inflammatory Crohn's disease and reflect disease location
}

Andrzej Wędrychowicz' ${ }^{1}$, Przemysław Tomasik ${ }^{2}$, Kinga Kowalska-Duplaga ${ }^{1}$, Stanisław Pieczarkowski ${ }^{1}$, Krzysztof Fyderek ${ }^{1}$

\author{
${ }^{1}$ Department of Paediatrics, Gastroenterology, and Nutrition, Jagiellonian University \\ Medical College, Krakow, Poland \\ ${ }^{2}$ Department of Clinical Biochemistry, Jagiellonian University Medical College, Krakow, \\ Poland
}

Submitted: 10 April 2021, Accepted: 1 June 2021

Online publication: 11 June 2021

Arch Med Sci 2021; 17 (4): 1114-1117

DOI: https://doi.org/10.5114/aoms/138349

Copyright @ 2021 Termedia \& Banach

\section{Abstract}

Introduction: The aim of our study was to assess antimicrobial peptides in children with Crohn's disease (CD).

Methods: Plasma elafin, cathelicidin, and $\alpha$ - and $\beta$-defensins were assessed in 35 children with CD using immunoassays. Phenotype and location of CD were assessed based on the results of endoscopic and radiological studies. Results: We found increased elafin, cathelicidin, and $\alpha$-defensins in children with inflammatory phenotype as compared to stricturing and penetrating phenotypes of CD. Additionally, we found increased elafin and cathelicidin in colonic location and $\alpha$-defensins in ileal CD locations.

Conclusions: Assessing antimicrobial peptides may be helpful in estimating of phenotype and location of CD lesions.

Key words: Crohn's disease, antimicrobial peptides, cathelicidin, elafin, $\alpha$ - and $\beta$-defensins.

Inflammatory bowel diseases (IBD), including ulcerative colitis (UC) and Crohn's disease (CD), are chronic inflammatory disorders of the gastrointestinal tract.

The theory of the pathogenesis of IBD suggest a loss of tolerance to intestinal microbiota and abnormal bacteria-host interactions that stimulate an autoimmunological response and upregulate inflammation in the gut $[1,2]$.

Antimicrobial peptides such as elafin, cathelicidin, and $\alpha$ - and $\beta$-defensins, are secreted by intestinal epithelial, immune, and Paneth cells of the gut and are important components of the innate host defence and mucosal barrier in the intestine [3, 4].

Alteration of the antimicrobial peptides may lead to changes in intestinal microbiome and induce the loss of host tolerance to microbiota [5]. The relationship between development of the gut inflammation during IBD and antimicrobial peptides concentrations is still unclear.

The aim of our study was to assess elafin, cathelicidin, $\alpha 1-3-$, and $\beta 1$-defensin concentrations in plasma and their relationship with the clinical presentation of CD in children.

\author{
Corresponding author: \\ Prof. Andrzej Wędrychowicz \\ Department of Paediatrics, \\ Gastroenterology, \\ and Nutrition \\ Jagiellonian University \\ Medical College \\ Krakow, Poland \\ Phone: +48 123339330 \\ E-mail: \\ miwedryc@cyf-kr.edu.pl
}


Table I. Clinical, demographic, and serological characteristics of the study groups

\begin{tabular}{|c|c|c|}
\hline Parameter & $\begin{array}{l}\text { CD group } \\
(n=35)\end{array}$ & $\begin{array}{l}\text { Control group } \\
(n=20)\end{array}$ \\
\hline Age of manifestation of disease symptoms (mean value and ranges) [years] & $13.8(6.5-18.0)$ & $13.5(6.0-18.0)$ \\
\hline Duration of symptoms (from onset to diagnosis, mean value and ranges) [weeks] & $18(2-20)$ & \\
\hline \multicolumn{3}{|l|}{ Clinical activity*: } \\
\hline Mild & $40 \%$ & \\
\hline Moderate & $37 \%$ & \\
\hline Severe & $23 \%$ & \\
\hline \multicolumn{3}{|l|}{ Serological testing: } \\
\hline ASCA positive & $75 \%$ & \\
\hline pANCA positive & $20 \%$ & \\
\hline Perianal lesions & $51 \%$ & \\
\hline Extraintestinal symptoms & $0 \%$ & \\
\hline \multicolumn{3}{|l|}{ CD phenotype: } \\
\hline Inflammatory (B1) & $74 \%$ & \\
\hline Stricturing (B2) & $9 \%$ & \\
\hline Penetrating (B3) & $17 \%$ & \\
\hline \multicolumn{3}{|l|}{ CD location: } \\
\hline Ileitis (L1) & $22 \%$ & \\
\hline Colitis (L2) & $29 \%$ & \\
\hline Ileo-colitis (L3) & $40 \%$ & \\
\hline Ileo-colono-gastro-duodenitis $(\mathrm{L} 3+\mathrm{L} 4 \mathrm{a})$ & $9 \%$ & \\
\hline
\end{tabular}

Methods. Thirty-five children with newly diagnosed CD (18 boys, 17 girls, mean age: 13.8 years, range: $6.5-18$ years) and 20 healthy children (10 boys, 10 girls, mean age; 13.5 years, range: 6.0-18.0 years) as controls were enrolled into the study. The clinical, demographic, and serological data of the CD patients and controls are summarized in Table I.

The antimicrobial peptides concentrations were measured using commercially available enzymelinked immunosorbent assay (ELISA) immunoassays produced by Hycult Biotechnology, Uden, the Netherlands for elafin, cathelicidin and $\alpha 1-3$-defensins, and those produced by LifeSpan BioSciences, Seattle, WA, USA for $\beta 1$-defensin.

Phenotype of CD lesions and their location in the gastrointestinal tract were assessed with upper endoscopy and ileo-colonoscopy with histopathological assessment of the mucosal specimens and radiology studies (ultrasound and magnetic resonance imaging), according to Paris CD classification [6].

All CD patients were newly diagnosed, in the active phase of the disease. None of the CD patients was previously treated with pharmacological or nutritional therapy, nor had previous surgical procedures before the enrolment in the study.

Statistical analysis was performed with Statistica 13.0 software (TIBCO Software Inc., Palo Alto,
CA, USA) using the Mann-Whitney $U$ test, Wilcoxon signed rank test, and Spearman's correlation rank test. $P<0.05$ was considered statistically significant. Antimicrobial peptide concentrations were expressed as median values and ranges.

The protocol of the study was approved by the Jagiellonian University Bioethical Committee (approval no. KBET/75/B/2009), and informed consent was obtained from all patients' legal guardians and all patients over 16 years of age at the time of enrolment in the study.

Results. We found significantly increased plasma elafin $(p<0.05)$, cathelicidin $(p<0.05)$, and $\alpha 1-3$ $(p<0.05)$ but not $\beta 1$-defensin concentrations in the subgroup of $C D$ children with inflammatory phenotype of $C D$ as compared to the subgroups of CD children with stricturing or penetrating phenotype or controls (Table II).

When we analysed antimicrobial peptides by different CD location, we found increased elafin and cathelicidin concentrations in colonic involvement (L2) compared to ileal (L1), ileo-colonic (L3), ileo-colono-gastro-duodenal involvement $(\mathrm{L} 3+\mathrm{L} 4 \mathrm{a})$ and controls $(p<0.05$, for all comparisons). In contrast, $\alpha 1-3$-defensins were increased in ileal (L1) and ileo-colono-gastro-duodenal $(\mathrm{L} 3+\mathrm{L} 4 \mathrm{a})$ involvement when compared to colonic (L2) $(p<0.05)$ and ileo-colonic (L3) $(p<0.05)$ involvement and controls $(p<0.05)$ (Table III). 
Table II. Antimicrobial peptide concentrations in subgroups of children with different phenotype of Crohn's disease (CD) and controls. Results are expressed as median values and ranges

\begin{tabular}{|lcccc|}
\hline Subgroup of CD & $\begin{array}{c}\text { Elafin } \\
{[\mathrm{ng} / \mathrm{ml}]}\end{array}$ & $\begin{array}{c}\text { Cathelicidin } \\
{[\mathrm{ng} / \mathrm{ml}]}\end{array}$ & $\begin{array}{c}\alpha 1-3-\text { defensins } \\
{[\mathrm{ng} / \mathrm{ml}]}\end{array}$ & $\begin{array}{c}\beta 1-\text { defensin } \\
{[\mathrm{pg} / \mathrm{ml}]}\end{array}$ \\
\hline Inflammatory (B1) & $15.6(11.6-50.1)^{\star}$ & $733(429.3-1102.9)^{\star}$ & $387(275.1-728.5)^{\star}$ & $201.9(150.3-281.1)$ \\
\hline Stricturing (B2) & $9.8(6.5-21.8)$ & $400(293.9-721.8)$ & $218(182.9-246.9)$ & $167.5(131.9-190.4)$ \\
\hline Penetrating (B3) & $1.46(1.21-11.4)$ & $323(142.3-480.2)$ & $243(196.2-529.1)$ & $152.7(115.2-161.4)$ \\
\hline Controls & $9.8(7.1-11.8)$ & $198.2(162.9-231.9)$ & $256.4(192.6-326.6)$ & $126.5(94.6-139.1)$ \\
\hline
\end{tabular}

${ }^{*} p<0.05$, when comparing inflammatory (B1) vs. stricturing (B2) and penetrating (B3) lesions and controls.

Table III. Antimicrobial peptide concentrations in children with different location of lesions in Crohn's disease (CD) and controls. Results are expressed as median values and ranges

\begin{tabular}{|lcccc|}
\hline $\begin{array}{l}\text { Location of CD } \\
\text { lesions }\end{array}$ & $\begin{array}{c}\text { Elafin } \\
{[\mathrm{ng} / \mathrm{ml}]}\end{array}$ & $\begin{array}{c}\text { Cathelicidin } \\
{[\mathrm{ng} / \mathrm{ml}]}\end{array}$ & $\begin{array}{c}\alpha 1-3-\text { defensins } \\
{[\mathrm{ng} / \mathrm{ml}]}\end{array}$ & $\begin{array}{c}\beta 1-\text { defensin } \\
{[\mathrm{pg} / \mathrm{ml}]}\end{array}$ \\
\hline Ileal (L1) & $12.4(8.1-15.2)$ & $464.4(372.4-580.1)^{\#}$ & $561.6(428.1-728.5)^{\wedge}$ & $209.3(161.4-273.6)$ \\
\hline Colonic (L2) & $39.8(31.2-50.1)^{\star}$ & $820.7(682.4-1102.9)^{\#}$ & $322.6(275.1-402.9)$ & $146.9(115.2-190.1)$ \\
\hline Ileo - colonic (L3) & $15.4(11.8-17.4)^{\star}$ & $599.7(420.9-760.8)^{\#}$ & $280.0(182.9-342.6)$ & $183.2(150.3-221.9)$ \\
\hline $\begin{array}{l}\text { Ileo - colono- gastro- } \\
\text { duodenal (L3 + L4a) }\end{array}$ & $7.7(1.21-10.4)$ & $310.4(142.9-350.8)$ & $420.8(340.9-529.1)^{\wedge}$ & $238.1(194.6-281.3)$ \\
\hline Controls & $9.8(7.1-11.8)$ & $198.2(162.9-231.9)$ & $256.4(192.6-326.6)$ & $126.5(94.6-139.1)$ \\
\hline
\end{tabular}

${ }^{*} p<0.05$, when compared colonic (L2) vs. ileal (L1), ileo-colonic (L3), ileo-colono-gastro-duodenal (L3 + L4a) lesions and controls and ileo-colonic (L3) vs. ileo-colono-gastro-duodenal $(L 3+\angle 4 a)$ and controls. ${ }^{*} p<0.05$, when compared colonic (L2) vs. ileal (L1), ileo-colonic (L3), ileo-colono-gastro-duodenal $(L 3+L 4 a)$ lesions and controls; ileo-colonic (L3) vs. ileal ( 1 1), ileo-colono-gastro-duodenal ( $33+L 4 a)$ and controls; ileal (L1) vs. ileo-colono-gastro-duodenal $(L 3+\angle 4 a)$ and controls. $\wedge p<0.05$, when compared ileal (L1) and ileo-colono-gastroduodenal $(L 3+L 4 a)$ vs. colonic $(L 2)$, ileo-colonic ( $L 3)$ lesions and controls.

There were no statistically significant differences among antimicrobial peptide concentrations in subgroups of patients with CD classified according to clinical, laboratory (C-reactive protein, erythrocyte sedimentation rate, calprotectin), and serological (pANCA or ASCA) parameters.

Discussion. The intestinal microbiome plays a crucial role in the pathogenesis of IBD. Aberrant and dysbiotic microbiota composition may lead to loss of normal regulatory immunological role of the microbiome and perpetuation of chronic inflammation.

Therefore, the interaction of host epithelial barrier dysfunction and dysbiotic microbiota in the gut may be responsible for the development of IBD [7].

Different inflammatory markers used in the diagnosis and assessment of CD activity did not reflect the phenotype and location of CD lesions [8].

Earlier studies on antimicrobial peptides showed conflicting results regarding their relationship with inflammatory lesions in IBD.

In our study, we showed increased plasma elafin concentrations in children with active inflammatory phenotype of $\mathrm{CD}$ lesions as compared to stricturing and penetrating ones, as well as in colonic location of inflammatory lesions compared to other locations. This observation confirmed earlier data in IBD patients $[9,10]$. However, it is impossible to compare the relationships between elafin and disease activity because no correlation and analysis of elafin and IBD activity were assessed in these studies.
Previous studies on cathelicidin in IBD showed conflicting results regarding their concentrations and relationship with inflammatory lesions.

Some authors reported no association between serum cathelicidin levels and the CD location and behaviour while the others showed significant negative correlation of serum cathelicidin with clinical activity of disease [11, 12].

Conversely, we showed increased cathelicidin in active $C D$ children with inflammatory lesions as compared to stricturing and penetrating lesions. Assessing the relationship between cathelicidin and the location of the inflammatory lesions, we showed the highest concentrations of cathelicidin in CD patients with colonic involvement when compared to CD patients with other locations involved.

Previously, research papers showed that $\alpha$-defensins were decreased in ileal mucosa of inflammatory lesions of adult CD patients, but in $C D$ children they were comparable to patients with colonic involvement and healthy children $[13,14]$.

Nevertheless, in later studies the authors found higher plasma levels of $\alpha$-defensins in active CD patients with ileal involvement when compared to inactive CD or control patients, which correlated with endoscopic severity and laboratory markers (CRP) [15].

In our study we found similar results. $\alpha 1-3$-defensins reached the highest levels in $C D$ patients with ileal involvement when compared to other locations of inflammatory lesions. Additionally, 
$\alpha$-1-3-defensins were elevated in CD children with inflammatory lesions as compared to stricturing and penetrating lesions.

Research data on $\beta$-defensins in IBD showed very conflicting results regarding their concentrations and relationship with inflammatory lesions [16].

In our study, we found $\beta 1$-defensins to be comparable in children with active $C D$ of different location in the gastro-intestinal tract and controls.

A major strength of this study was that it was the first study to report relationships among antimicrobial peptides and phenotypes of CD in children.

Another strength of our study was that we focused only on newly diagnosed CD patients in the initial period of treatment. Thus, we assessed antimicrobial peptide concentrations only at 2 points: at the baseline, before the induction therapy, and after 2 weeks of treatment. We analysed the activity and location of CD in detail (clinical, endoscopic and histopathological activity, and laboratory parameters) at the beginning of the treatment.

The main limitations of our study were the relatively small group of patients and short follow-up during the treatment. The reason for the relatively short follow-up period was that we wanted to eliminate the influence of the different concomitant treatments on the antimicrobial peptide concentrations, which meant resulted that we assessed antimicrobial peptides only in children with $C D$ treated with exclusive enteral nutrition therapy and 5-amino salicylates.

Our results showed significantly elevated plasma elafin, cathelicidin, and $\alpha 1-3$ - but not $\beta 1$-defensin concentrations in children with inflammatory phenotype of CD. Additionally, we found the highest increase of plasma elafin and cathelicidin in patients with colonic location of CD lesions (L2), while plasma $\alpha 1-3$-defensins reached the highest concentrations in patients with ileal involvement.

In conclusion, assessment of plasma elafin, cathelicidin, and $\alpha 1-3$-defensins may be helpful in estimation of the phenotype and location of $C D$ lesions in children. Future research studies on larger groups of patients are necessary to confirm our findings. Additionally, simultaneous assessment of antimicrobial peptides and intestinal microbiome could be of value to establish possible effect of dysbiotic microbiome on antimicrobial peptides and the correlation of both with the phenotype of $C D$ in children.

\section{Conflict of interest}

The authors declare no conflict of interest.

\section{References}

1. Nishida A, Inoue R, Inatomi O, Bamba S, Naito Y, Andoh A. Gut microbiota in the pathogenesis of inflammatory bowel disease. Clin J Gastroenterol 2018; 11: 1-10.
2. Kowalska-Duplaga K, Gosiewski T, Kapusta P, et al. Differences in the intestinal microbiome of healthy children and patients with newly diagnosed Crohn's disease. Sci Rep 2019; 9: 18880.

3. Marks DJ, Segal AW. Innate immunity in inflammatory bowel disease: a disease hypothesis. J Pathol 2008; 214: 260-6.

4. Hirata Y, Ihara S, Koike K. Targeting the complex interactions between microbiota, host epithelial and immune cells in inflammatory bowel disease. Pharmacol Res 2016; 113: 574-84.

5. Wehkamp J, Koslowski M, Wang G, Stange EF. Barrier dysfunction due to distinct defensin deficiencies in small intestinal and colonic Crohn's disease. Mucosal Immunol 2008; 1 Suppl 1: S67-74.

6. Levine A, Griffiths A, Markowitz J, et al. Pediatric modification of the Montreal classification for inflammatory bowel disease: the Paris classification. Inflamm Bowel Dis 2011; 17: 1314-21.

7. Satokari R. Contentious host-microbiota relationship in inflammatory bowel disease: can foes become friends again? Scand I Gastroenterol 2015; 50: 34-42.

8. Słowińska-Solnica K, Pawlica-Gosiewska D, Gawlik K, et al. Serum inflammatory markers in the diagnosis and assessment of Crohn's disease activity. Arch Med Sci 2021; 17: 252-7.

9. Kim JM. Antimicrobial proteins in intestine and inflammatory bowel diseases. Intest Res 2014; 12: 20-33.

10. Schmid M, Fellermann K, Fritz P, Wiedow O, Stange EF, Wehkamp J. Attenuated induction of epithelial and leukocyte serine antiproteases elafin and secretory leukocyte protease inhibitor in Crohn's disease. J Leukoc Biol 2007; 81: 907-15.

11. Sun L, Wang W, Xiao W, Yang H. The roles of cathelicidin LL-37 in inflammatory bowel disease. Inflamm Bowel Dis 2016; 22: 1986-91.

12. Tran DH, Wang J, Ha C, et al. Circulating cathelicidin levels correlate with mucosal disease activity in ulcerative colitis, risk of intestinal stricture in Crohn's disease, and clinical prognosis in inflammatory bowel disease. BMC Gastroenterol 2017; 17: 63 .

13. Zilbauer M, Jenke A, Wenzel G, et al. Intestinal alpha-defensin expression in pediatric inflammatory bowel disease. Inflamm Bowel Dis 2011; 17: 2076-86.

14. Wehkamp J, Salzman NH, Porter E, et al. Reduced Paneth cell alpha-defensins in ileal Crohn's disease. Proc Natl Acad Sci USA 2005; 102: 18129-34.

15. Cerrillo E, Moret I, Iborra M, et al. Alpha-defensins $(\alpha$-Defs) in Crohn's disease: decrease of ileal $\alpha$-Def 5 via permanent methylation and increase in plasma $\alpha$-Def 1-3 concentrations offering biomarker utility. Clin Exp Immunol 2018; 192: 120-8.

16. Wehkamp J, Harder J, Weichenthal M, et al. Inducible and constitutive beta-defensins are differentially expressed in Crohn's disease and ulcerative colitis. Inflamm Bowel Dis 2003; 9: 215-23. 